\title{
A Single Motivational Lecture Can Promote Modest Weight Loss: A Randomized Controlled Trial
}

\author{
Yoshio Nakata ${ }^{a}$ Hiroyuki Sasai $^{b}$ Takehiko Tsujimoto ${ }^{c}$ \\ Koichi Hashimoto $^{d}$ Hiroyuki Kobayashi $^{d}$ \\ ${ }^{\text {a }}$ Faculty of Health and Sport Sciences, University of Tsukuba, Tsukuba, Japan; \\ ${ }^{b}$ Graduate School of Arts and Sciences, The University of Tokyo, Tokyo, Japan; \\ 'Faculty of Human Sciences, Shimane University, Matsue, Japan; ${ }^{\mathrm{d}}$ Faculty of Medicine, \\ University of Tsukuba, Tsukuba, Japan
}

\section{Keywords}

Body weight changes - Diet - Exercise $\cdot$ Health education · Obesity management

\begin{abstract}
Background: Obesity is a public health problem worldwide. To widely disseminate weightloss interventions across the target population, a cost-effective approach is needed. Objective: We aimed to test whether a single motivational lecture could promote weight loss. Methods: Our study was a 3-month randomized controlled trial, and we recruited participants via local newspaper advertisements in 3 cities in Ibaraki Prefecture, Japan, and randomly assigned them to a control group (no intervention) and an intervention group, who attended a single motivational lecture lasting approximately $2 \mathrm{~h}$. No other lectures or textbooks were provided. The eligibility criteria included an age of 40-64 years, a body mass index (BMI) of 25-40 kg/ $\mathrm{m}^{2}$, and the presence of at least 1 component of metabolic syndrome. The primary outcome was body weight change at 3 months. Results: We enrolled 145 eligible participants with a mean age of $53.8 \pm 7.1$ years and a BMI of $28.5 \pm 3.1 \mathrm{~kg} / \mathrm{m}^{2}$. The 3 -month body weight change in the control and intervention groups was $-0.65 \mathrm{~kg}$ (95\% confidence interval $[\mathrm{Cl}]-1.09$ to $-0.20)$ and $-2.48 \mathrm{~kg}(95 \% \mathrm{Cl}-3.01$ to -1.95$)$, respectively. The between-group difference was $1.83 \mathrm{~kg}(95 \% \mathrm{Cl} 1.15-2.51)$. Conclusions: The significant difference suggested that a single motivational lecture is an effective option to promote modest weight loss in the short term. Registry (UMIN000010505) on April 16, 2013. 
Nakata et al.: Effect of a Single Motivational Lecture on Weight Loss

\section{Introduction}

Obesity is a public health problem worldwide. Global mean body mass index (BMI) increased from $21.7 \mathrm{~kg} / \mathrm{m}^{2}$ in 1975 to $24.2 \mathrm{~kg} / \mathrm{m}^{2}$ in 2014 in men and from $22.1 \mathrm{~kg} / \mathrm{m}^{2}$ to 24.4 $\mathrm{kg} / \mathrm{m}^{2}$ in women, respectively [1]. Even in Japan, the proportion of obesity (judged as BMI $\geq 25 \mathrm{~kg} / \mathrm{m}^{2}$ ) has been steadily increasing, especially in men [2]. To tackle this problem, the Japanese Ministry of Health, Labor, and Welfare launched a nationwide health check-up and interventional program for targeting metabolic syndrome in April 2008 [3]. The interventional program consists of motivational counseling and a face-to-face or Internet-based intervention. Although several types of intervention programs have been implemented, most are time-, labor-, and cost-intensive. To widely disseminate weight loss intervention across overweight and obese populations, a cost-effective approach is needed.

A brief intervention is a potential option to disseminate weight loss interventions to the public. Aveyard et al. [4] examined whether a brief intervention by a physician is effective for reducing body weight in patients with obesity; the intervention was a 30-s referral to a weight management program. A significant effect on body weight at 12 months was observed (an adjusted difference of $1.43 \mathrm{~kg}$ between the intervention group and the control group). Beeken et al. [5] tested the effects of a brief intervention using Ten Top Tips, a leaflet based on the habit-formation theory. People in the intervention arm received the leaflet and attended a single 30 -min session. At 3 months, a significant difference $(0.87 \mathrm{~kg})$ in body weight was observed. Similar effects were also observed in the control groups of brief interventions. A systematic review and meta-analysis of weight change among people randomized to minimal intervention control groups showed a significant weight loss of $0.8 \mathrm{~kg}$ at 12 months [6].

In our previous randomized controlled trial (RCT) [7, 8], we tested the effects of providing educational materials such as textbooks and diaries and group-based support with a 2-h single motivational lecture for the control group. Weight loss at 6 months in the control group, educational group, and group-based support group was $2.9,4.7$, and $7.7 \mathrm{~kg}$, respectively. This RCT provided evidence that both educational materials and group-based support are effective components in a weight loss program and suggested that just a single 2-h motivational lecture has the potential to induce significant weight loss. If a single motivational lecture is effective in promoting weight loss, it could be a potentially cost-effective approach.

Here, we aimed to examine the effectiveness of a 2-h single motivational lecture in achieving weight loss compared with no intervention as a control. In the weight loss intervention trials, the characteristics of participants who were willing and motivated might have influenced the trial results [9-11] and caused the Hawthorne effect. We thus set a no-intervention control group and tried to demonstrate the effectiveness of a single motivational lecture on weight loss in a 3-month RCT.

\section{Materials and Methods}

\section{Design, Participants, and Randomization}

This study involved a 3-month parallel-group RCT. We conducted our trial at community centers in 3 cities (Tsukuba, Mito, and Chikusei) in the Ibaraki Prefecture, Japan, between June 2013 and June 2014. This article adheres to the Consolidated Standards of Reporting Trials (CONSORT) 2010 guidelines [12].

Participants were recruited via local newspaper advertisements in the 3 cities. The participants were included in the study if they met the following criteria: an age of 40-64 years, a BMI of $25-40 \mathrm{~kg} / \mathrm{m}^{2}$, and the presence of at least 1 component of the metabolic syndrome (abdominal obesity, hypertension, dyslipidemia, or hyperglycemia) $[3,13,14]$. The 
Nakata et al.: Effect of a Single Motivational Lecture on Weight Loss

Table 1. Description of presentation slides used in the single motivational lecture

\begin{tabular}{|c|c|}
\hline Slide No. & Theme/topic \\
\hline 1 & Title slide \\
\hline 2 & $\begin{array}{l}\text { Body mass index (BMI) calculation and its Japanese and global classifications of lean, normal, } \\
\text { overweight, and obesity }\end{array}$ \\
\hline 3 & Prevalence of obesity among US adults \\
\hline 4 & Prevalence of obesity among Japanese adults \\
\hline 5 & Scientific evidence for determining obesity as a BMI of $\geq 25$ for Japanese adults [13] \\
\hline 6 & 30-year trend in obesity prevalence by sex in Japan \\
\hline 7 & Subcutaneous- or visceral-type obesity \\
\hline 8 & $\begin{array}{l}\text { Scientific evidence for determining visceral-type obesity as abdominal visceral fat area of } \\
\geq 100 \mathrm{~cm}^{2} \text { for Japanese adults [13] }\end{array}$ \\
\hline 9 & Close relationship between waist circumference and visceral fat area [14] \\
\hline 10 & Diagnostic criteria for metabolic syndrome in Japan \\
\hline 11 & Clustering of cardiometabolic risks and cardiovascular mortality [17] \\
\hline 12 & Clustering of cardiometabolic risks and ischemic heart disease [18] \\
\hline 13 & Clustering of cardiometabolic risks and stroke [18] \\
\hline 14 & Weight loss amount and improvement rate in metabolic syndrome components [15] \\
\hline 15 & Short- and long-term goals for weight loss to improve metabolic syndrome \\
\hline 16 & Graphical representation of 1-, 2-, and 3-kg body fat \\
\hline 17 & Basic principle to lose weight \\
\hline $18-21$ & How to reduce your dietary intake \\
\hline $22-24$ & How to increase your energy expenditure by physical activity \\
\hline 25 & Basic regimen for a weight loss program \\
\hline 26 & Graphical representation of body fats and their equivalent amount of energy \\
\hline 27 & What we need for successful weight management \\
\hline 28 & Importance of drinking water \\
\hline 29 & Importance of weigh-ins \\
\hline 30 & Practical recommendations for successful weight management \\
\hline 31 & Summary and take-home messages \\
\hline
\end{tabular}

criteria for each metabolic syndrome component complied with the Japanese nationwide mandatory health check-up program [3]. Participants were ineligible if they had a history of diabetes, coronary disease, or stroke; were pregnant or planned to become pregnant; or had participated in another weight loss program during the previous 6 months. Participants whose cohabitant(s) were enrolled in this study were also excluded.

After the initial eligibility assessment, eligible participants were randomly assigned (1: 1) into a control or intervention group with stratification by city and sex using simple computerized randomization procedures. A biostatistician who had no contact with the participants or trial staff generated the group allocation code which was concealed at a secure central location until eligible participants were finalized.

The participants in the control group did not receive any intervention for 3 months; however, they attended measurement sessions at baseline and after 3 months. Participants in the intervention group received a single motivational lecture held 1 or 2 weeks after the baseline visit. No financial compensation was offered to any of the participants in the 3-month study period. After the collection of data, all participants could participate in our verified 3-month weight loss program $[7,15]$, which included textbooks, notebooks, and group-based support sessions.

\section{The Motivational Lecture}

The intervention participants received a 2-h, group-based, single motivational lecture held in each city and delivered by an investigator (Y.N.). The structure and slide-by-slide description of the lecture is given in Table 1 . Briefly, the lecture comprised an introduction to 
Nakata et al.: Effect of a Single Motivational Lecture on Weight Loss

the Japanese nationwide health check-up and intervention program initiated in April 2008 that specifically targets metabolic syndrome $[3,13,14]$, clustered cardiovascular risk factors, serious outcomes [16-18], and the target value for alleviating metabolic syndrome [15]. The participants were also given typical weight management recommendations about diet and exercise as well as other behavioral modifications. The recommendations involved an energyrestricted diet of 1,200 kcal/day for women and 1,600 kcal/day for men [15, 19, 20] and a minimum of $1,000 \mathrm{kcal} /$ week of physical activity [21]. However, each target weight and behavior objective in terms of diet and physical activity was set by the participants themselves. All participants were encouraged to weigh themselves daily.

\section{Measurements}

The measurement visits were conducted at baseline and after 3 months. The primary outcome was a 3-month change in body weight. Secondary outcomes included a change in waist circumference, systolic and diastolic blood pressure, and the level of triglycerides, highdensity lipoprotein (HDL) cholesterol, and fasting plasma glucose. Total energy intake and physical activity were also assessed as compliance measures. All study outcomes were measured by trained staff members, as described below. Basic characteristics, such as demographic, socioeconomic, and lifestyle variables as well as treatment and weight control status were recorded only at baseline. As possible predictors of weight change, the stage of change and self-efficacy for lifestyle modifications were also assessed at baseline, as described below.

Basic Characteristics

Using a self-administered questionnaire, participants reported their age, sex, working status (full-time or not), educational attainment (4-year college graduate or not), household income ( $\geq$ JPY 5,000,000/year or not), and marital status (currently married or not). They provided information on smoking (never-smoker or not) and drinking (current drinker or not) status, and whether they were currently being treated for hypertension and dyslipidemia (Yes or No). Participants also reported their lifetime frequency of intentional weight loss ( $\geq 4$ times or not), where weight loss was defined as a loss of $\geq 3 \mathrm{~kg}$.

Stage of Change for Lifestyle Modification

According to the transtheoretical model of behavior change [22], the stage of change for lifestyle modification was defined as the degree of readiness to change lifestyle behavior to reduce body weight. Participants responded to a single question of "Are you trying to modify your lifestyle behaviors to lose body weight?" by choosing one of the following responses: (1) "Currently, I do not try to modify my lifestyle behaviors to lose weight, and I have no intention of modifying it in the future" (precontemplation); (2) "I am thinking about modifying my lifestyle behaviors to lose weight in the near future (within 6 months)" (contemplation); (3) "I modify my lifestyle behaviors to lose weight, but not regularly" (preparation); (4) "I currently modify my lifestyle behaviors to lose weight, but I only started it within the past 6 months" (action); and (5) "I currently modify my lifestyle behaviors to lose weight regularly, and have continued it for more than 6 months" (maintenance).

Self-Efficacy for Weight Loss

Self-efficacy for weight loss was defined as the degree of confidence regarding participants' capability to modify their dietary and activity behaviors for weight loss. Latimer et al. [23] developed the Physical Activity and Nutrition Self-Efficacy (PANSE) scale. To use this scale in our study, we prepared a Japanese translation and modified some sections to provide more specific examples of food items appropriate for the Japanese population. The PANSE is an 11-item scale divided into dietary behavior ( 8 items) and activity behavior (3 items) 
Nakata et al.: Effect of a Single Motivational Lecture on Weight Loss

subscales. Participants responded about how confident they felt about specific behaviors (e.g., reducing portion size at meals or snacks) on a scale of 1 (not at all) to 9 (completely). Responses were summarized into subtotal scores for dietary (range 8-72) and activity (range 3-27) behaviors, with higher scores indicating better self-efficacy in losing weight.

Anthropometrics

Height was assessed with a precision of $0.1 \mathrm{~cm}$ using a portable stadiometer (213; Seca, Hamburg, Germany), and body weight was measured with a precision of $0.05 \mathrm{~kg}$ using a calibrated digital scale (WB-150; Tanita, Tokyo, Japan). The participants' BMI was calculated. Waist circumference was measured twice with a precision of $0.1 \mathrm{~cm}$ at the level of the umbilicus using a flexible plastic tape while the participant stood naturally; this was performed in line with the standard guidelines of the Japanese nationwide health check-up program [3]. The average value of the 2 measurements was used for data analysis.

Metabolic Syndrome Components

The participants were seated at rest for $\geq 5$ min with their arms placed at heart level. Systolic and diastolic blood pressures were then measured twice using an automated sphygmomanometer (HEM-7430; Omron Healthcare, Kyoto, Japan). The average value of the 2 readings was incorporated into the data analysis. A venous blood sample was drawn from each participant after an overnight fast of $\geq 12 \mathrm{~h}$. Serum triglyceride levels were tested enzymatically (Determiner L TG II; Kyowa Medex, Tokyo, Japan). Serum HDL cholesterol was determined using the selective inhibition method (MetaboLead HDL-C; Kyowa Medex). Blood glucose was measured using the hexokinase-G-6-phosphate dehydrogenase method (L-Type Glu 2; Wako Pure Chemical Industries, Osaka, Japan). An independent laboratory (Kotobiken Medical Laboratories, Ibaraki, Japan) assayed all blood samples.

Dietary Intake

Total energy intake was measured using 3-day weighed food records and was used as a reference for dietary intake [24]. The participants recorded all food items they had consumed over 3 days, including 2 weekdays and 1 weekend day. They quantified the food items using standard measuring cups, spoons, and digital scales. To ensure overall comparability, an experienced nutritionist, blinded to the group allocation, reviewed and analyzed all food records using a computer program (Eiyoukun, Kenpakusya v6.0, Tokyo, Japan) with the Japanese food composition tables (2010 edition).

Physical Activity

The participants were fitted with a triaxial accelerometer (Active style Pro HJA-350IT; Omron Healthcare) at the waist for 14 consecutive days. The accelerometer counted their daily steps and estimated the intensity of physical activity (expressed as metabolic equivalents [METs]) using a validated algorithm $[25,26]$. They were instructed to detach the devices when sleeping, undertaking a water-based activity (e.g., bathing or swimming), or participating in contact sports (e.g., soccer or rugby) for safety reasons. A valid day was defined as a wear time of $\geq 10 \mathrm{~h}$ [27]. If no acceleration signals over a 1-min time interval lasted for $\geq 60$ consecutive min, the period was assumed as "non-wear" [28]. When valid data were available for $\geq 3$ days, the average daily step count and time spent in moderate-to-vigorous physical activity (MVPA; $\geq 3$ METs) were calculated for each participant [28].

\section{Statistical Analysis}

All data were handled and analyzed in full accordance with the analysis plan in the study protocol using the open-source statistical environment $R$ (v3.4.4 for Windows 64-bit). All 
Nakata et al.: Effect of a Single Motivational Lecture on Weight Loss

Fig. 1. Participant flowchart. ITT, intention-to-treat.



$p$ values $<0.05$ were considered statistically significant. Baseline characteristics were summarized as mean \pm SD for continuous variables and $n(\%)$ for categorical variables.

The required sample size, with a $1.5 \pm 3.0 \mathrm{~kg}$ difference in weight change between the groups based on our previous RCT [7], an $\alpha$ level of 5\%, and a power of $80 \%$, was 128 participants. Assuming an eligibility rate of $90 \%$, the final required sample size was determined at 150 participants.

Our primary analysis conformed to an intention-to-treat (ITT) principle, with missing data replaced by baseline observations carried forward. Primary, secondary, and compliance outcomes are presented as a mean and 95\% confidence interval (CI). The unpaired Student $t$ test examined the statistical significance of between-group differences. The $\chi^{2}$ test was performed to examine the between-group difference in categorical variables. We also computed between-group differences for weight change and $\geq 3 \%$ or $\geq 5 \%$ weight loss after adjusting for baseline weight, smoking status, and baseline MVPA by using multiple regression or logistic regression analyses.

The secondary analyses explored possible predictors for weight change using data from participants with no missing data. Multiple logistic regression models were established with a $\geq 3 \%$ or $\geq 5 \%$ weight loss as a dependent variable; the group assignment (intervention group), age (every 1 year), sex (female), smoking status (never-smoker), marital status (currently married), working status (full-time worker), educational attainment (4-year college graduate), annual household income ( $\geq$ JPY 5,000,000), stage of change for lifestyle modification (every stage), self-efficacy subscales for dietary and activity behaviors (every 5 points for both), and baseline MVPA (every $10 \mathrm{~min} /$ day) were included as potential predictors. The analyses were repeated as described but only for the participants in the intervention group.

\section{Results}

Figure 1 shows the participant flowchart. After recruitment, 170 candidates gave written informed consent at the initial introductory sessions held in the 3 cities. Of these, 157 were assessed for eligibility and 145 (92.4\%) fulfilled the eligibility criteria. The participants were 
Nakata et al.: Effect of a Single Motivational Lecture on Weight Loss

Table 2. Baseline participant characteristics of 145 participants

\begin{tabular}{|c|c|c|}
\hline & $\begin{array}{l}\text { Control group } \\
(n=72)\end{array}$ & $\begin{array}{l}\text { Intervention group } \\
(n=73)\end{array}$ \\
\hline \multicolumn{3}{|l|}{ Demographic and socioeconomic status } \\
\hline Age, years & $53.0 \pm 6.9$ & $54.5 \pm 7.3$ \\
\hline Female sex & $58(80.6)$ & $56(76.7)$ \\
\hline Currently married & $61(84.7)$ & $64(88.9)$ \\
\hline Full-time worker & $28(38.9)$ & $24(33.3)$ \\
\hline Four-year college graduate & $13(18.1)$ & $13(18.1)$ \\
\hline Household income $\geq$ JPY 5,000,000 & $40(55.6)$ & $46(63.9)$ \\
\hline \multicolumn{3}{|l|}{ Lifestyle, and treatment status } \\
\hline Never-smoker & $48(66.7)$ & $57(78.1)$ \\
\hline Current drinker & $40(55.6)$ & $40(54.8)$ \\
\hline Treatment for hypertension & $29(40.3)$ & $26(35.6)$ \\
\hline Treatment for dyslipidemia & $18(25.0)$ & $20(27.4)$ \\
\hline Weight loss attempts, $\geq 4$ times & $10(13.9)$ & $10(13.9)$ \\
\hline \multicolumn{3}{|l|}{ Stage of change for lifestyle modification } \\
\hline Precontemplation & $0(0)$ & $0(0)$ \\
\hline Contemplation & $22(30.6)$ & $19(26.0)$ \\
\hline Preparation & $39(54.2)$ & $41(56.2)$ \\
\hline Action & $8(11.1)$ & $8(11.0)$ \\
\hline Maintenance & $3(4.2)$ & $5(6.8)$ \\
\hline \multicolumn{3}{|l|}{ Self-efficacy for weight loss } \\
\hline Dietary behaviors, scale range 8-72 & $56.4 \pm 6.5$ & $56.4 \pm 7.5$ \\
\hline Activity behaviors, scale range $3-27$ & $17.2 \pm 3.9$ & $18.5 \pm 3.9$ \\
\hline \multicolumn{3}{|l|}{ Anthropometrics } \\
\hline Height, cm & $160.4 \pm 7.1$ & $158.9 \pm 7.8$ \\
\hline Weight, kg & $74.2 \pm 10.3$ & $71.6 \pm 10.5$ \\
\hline Body mass index, $\mathrm{kg} / \mathrm{m}^{2}$ & $28.8 \pm 3.1$ & $28.3 \pm 3.0$ \\
\hline Waist circumference, $\mathrm{cm}$ & $99.4 \pm 8.2$ & $97.3 \pm 8.0$ \\
\hline \multicolumn{3}{|l|}{ Metabolic syndrome component } \\
\hline Systolic blood pressure, $\mathrm{mm} \mathrm{Hg}$ & $139.3 \pm 16.3$ & $138.5 \pm 16.6$ \\
\hline Diastolic blood pressure, $\mathrm{mm} \mathrm{Hg}$ & $86.6 \pm 9.6$ & $84.9 \pm 12.8$ \\
\hline Triglycerides, mg/dL & $127.3 \pm 60.0$ & $126.0 \pm 56.3$ \\
\hline HDL cholesterol, mg/dL & $56.9 \pm 12.0$ & $58.8 \pm 13.9$ \\
\hline Fasting plasma glucose, $\mathrm{mg} / \mathrm{dL}$ & $99.7 \pm 24.0$ & $98.8 \pm 12.6$ \\
\hline \multicolumn{3}{|l|}{ Energy intake and physical activity } \\
\hline Total energy intake, kcal/day & $1,996 \pm 431$ & $1,941 \pm 467$ \\
\hline Step count ${ }^{\mathrm{a}}$, steps/day & $5,805 \pm 2,394$ & $6,933 \pm 2,696$ \\
\hline $\mathrm{MVPA}^{\mathrm{a}}$, min/day & $56.6 \pm 29.3$ & $71.5 \pm 34.2$ \\
\hline
\end{tabular}

Data are expressed as mean \pm SD or $n(\%)$. HDL, high-density lipoprotein; MVPA, moderate-to-vigorous physical activity.

a Valid data were collected for 143 participants.

randomized into the control $(n=72)$ and intervention $(n=73)$ groups and served in the primary ITT analyses later. There were no notable differences in baseline characteristics, except for step count and MVPA, between the groups (Table 2). The intervention participants were significantly more active than the control participants at baseline $(p<0.05)$. Of the 145 randomized participants, $68(94.4 \%)$ in the control group and 70 (95.9\%) in the intervention group attended the 3-month follow-up visit. The retention rate did not differ by group 
Table 3. Changes in body weight and metabolic syndrome components over 3 months $(n=145)$

\begin{tabular}{|c|c|c|c|}
\hline Characteristic & Control group $(n=72)$ & Intervention group $(n=73)$ & $p$ value \\
\hline \multicolumn{4}{|l|}{ Anthropometrics } \\
\hline Weight, kg & $-0.65(-1.09$ to -0.20$)$ & $-2.48(-3.01$ to -1.95$)$ & $<0.001$ \\
\hline Weight loss $\geq 3 \%$ & $12(16.7)$ & $42(57.5)$ & $<0.001$ \\
\hline Weight loss $\geq 5 \%$ & $4(5.6)$ & $19(26.0)$ & 0.002 \\
\hline Body mass index & $-0.25(-0.41$ to -0.08$)$ & $-0.99(-1.19$ to -0.79$)$ & $<0.001$ \\
\hline Waist circumference, $\mathrm{cm}$ & $-1.14(-1.67$ to -0.60$)$ & $-2.72(-3.35$ to -2.09$)$ & $<0.001$ \\
\hline \multicolumn{4}{|l|}{ Metabolic syndrome component } \\
\hline Systolic blood pressure, $\mathrm{mm} \mathrm{Hg}$ & $-2.51(-5.16$ to 0.15$)$ & $-3.36(-6.30$ to -0.42$)$ & 0.67 \\
\hline Diastolic blood pressure, mm Hg & $-1.18(-2.94$ to 0.58$)$ & $-1.48(-3.23$ to 0.27$)$ & 0.81 \\
\hline Triglycerides, mg/dL & $-11.2(-26.5$ to 4.0$)$ & $-17.0(-27.5$ to -6.6$)$ & 0.54 \\
\hline HDL cholesterol, mg/dL & $0.51(-1.07$ to 2.10$)$ & $0.56(-0.87$ to 1.99$)$ & 0.96 \\
\hline Fasting plasma glucose, $\mathrm{mg} / \mathrm{dL}$ & $-2.49(-7.34$ to 2.37$)$ & $-3.93(-5.87$ to -1.99$)$ & 0.58 \\
\hline \multicolumn{4}{|l|}{ Energy intake and physical activity } \\
\hline Total energy intake, kcal/day & $-116.5(-218.7$ to -14.3$)$ & $-183.3(-277.8$ to -88.8$)$ & 0.34 \\
\hline Step count ${ }^{\mathrm{a}}$, steps/day & $-13.5(-414.1$ to 387.1$)$ & $607.4(-41.3$ to 1256.1$)$ & 0.11 \\
\hline $\mathrm{MVPA}^{\mathrm{a}}$, min/day & $3.70(-0.56$ to 7.96$)$ & $4.17(-2.13$ to 10.47$)$ & 0.90 \\
\hline
\end{tabular}

Data are expressed as mean (95\% confidence interval) or $n(\%)$.

${ }^{a}$ Valid data were collected for 143 participants. HDL, high-density lipoprotein; MVPA, moderate-to-vigorous physical activity.

assignment $(p=0.68)$. The missing observations were mostly due to personal or medical reasons unrelated to the study. There were no clinically significant adverse events that were judged by the investigators to be related to participation in the trial.

Table 3 shows the ITT analyses of the primary, secondary, and compliance outcomes. The 3-month body weight change in the control and intervention groups was $-0.65 \mathrm{~kg}$ (95\% CI -1.09 to -0.20$)$ and $-2.48 \mathrm{~kg}(95 \% \mathrm{CI}-3.01$ to -1.95$)$, respectively. The betweengroup difference was $1.83 \mathrm{~kg}$ (95\% CI 1.15-2.51). The proportion of participants who achieved a weight loss of $\geq 3 \%$ or $\geq 5 \%$ was significantly higher in the intervention group than in the control group $(p<0.05)$. After adjusting for baseline weight, smoking status, and baseline MVPA, the between-group difference in weight change was $-2.07 \mathrm{~kg}(95 \% \mathrm{CI}$ -2.78 to -1.37 ), and the odds ratio for the intervention group in achieving a $\geq 3 \%$ or $\geq 5 \%$ weight loss was 8.16 (95\% CI 3.57-18.63) and 6.34 (95\% CI 1.98-20.29), respectively. The reduction in waist circumference was also significantly greater in the intervention group than in the control group $(p<0.05)$. Although the remaining outcomes tended to show a greater improvement in the intervention group, no improvements differed significantly between the 2 groups.

Table 4 uses the data from participants who completed the study with no missing data ( $n=138$ ) to explore potential predictors for a weight loss of $\geq 3 \%$ or $\geq 5 \%$ using logistic regression analysis. Belonging to the intervention group, having a higher baseline self-efficacy for activity behavior and a lower baseline MVPA level were all significantly associated with achieving a weight loss of $\geq 3 \%(p<0.05)$. In addition, assignment to the intervention group was the only variable that was significantly associated with a weight loss of $\geq 5 \%(p<0.05)$. When the same analyses were repeated with restriction of the analytic sample to the intervention participants $(n=70)$, a higher baseline self-efficacy for activity behavior and a lower baseline MVPA level were both significantly associated with achieving a weight loss of $\geq 3 \%$ $(p<0.05)$. However, none of the characteristics predicted a weight loss of $\geq 5 \%$ in the intervention group. 
Nakata et al.: Effect of a Single Motivational Lecture on Weight Loss

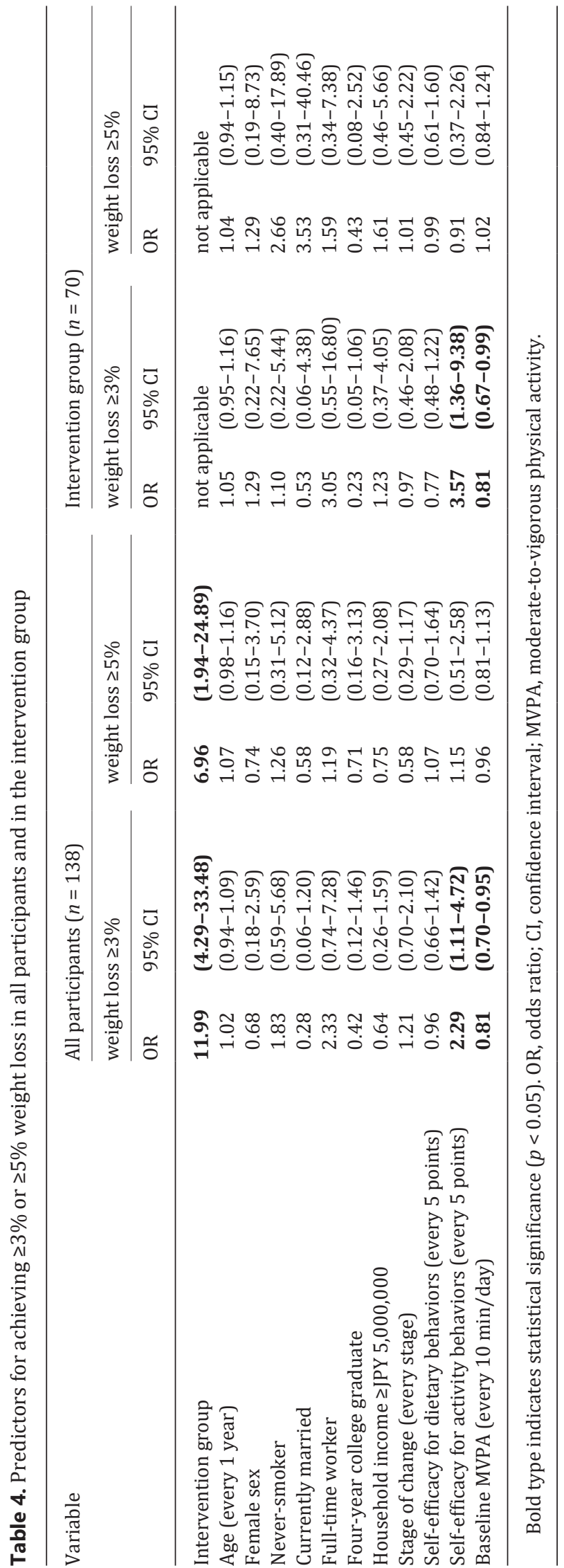


Nakata et al.: Effect of a Single Motivational Lecture on Weight Loss

\section{Discussion}

In this study, we examined the effectiveness of a 2-h single motivational lecture compared with no intervention as a control. Our 3-month RCT demonstrated the effectiveness of a single lecture to promote modest weight loss (crude: $1.83 \mathrm{~kg}$ and adjusted: $2.07 \mathrm{~kg}$ ). Subsequent exploratory logistic regression analyses revealed that a higher baseline self-efficacy for activity behavior and a lower baseline MVPA level were associated with achieving $\geq 3 \%$ weight loss. Assignment to the intervention group was the only significant predictor of $\geq 5 \%$ weight loss.

A brief intervention, such as the single motivational lecture used in this study, is a costeffective option to achieve modest weight loss. Aveyard et al. [4] showed the effectiveness of a 30-s referral to a weight management program by physicians in primary care. This brief opportunistic intervention motivated patients with obesity to participate in a weight loss program. Commercial weight loss programs have been proven to be more effective and cheaper than general one-to-one counseling in primary care [29]. Another brief intervention is Ten Top Tips, used by Beeken et al. [5] who showed that a single 30-min session with the leaflet resulted in a significant difference in body weight $(0.87 \mathrm{~kg})$ compared with a usual-care control group at 3 months. A meta-analysis [6] showed that people randomized to minimal intervention control groups could lose $0.8 \mathrm{~kg}(95 \% \mathrm{CI} 0.4-1.1)$ at 12 months. The control interventions were categorized as offering leaflets only, a single session of advice, or $>1$ session of advice from someone without specialist skills in supporting weight loss. In an unadjusted model, a more intense intervention was associated with additional weight loss. The number of weighins in the study period was also associated with additional weight loss; however, in the adjusted model using 2 variables, neither of these associations was found. Therefore, there is a lack of evidence that brief interventions and regular weighing promote additional weight loss.

In our study, we gave a 2-h single motivational lecture to the participants. There were 2 opportunities for weigh-ins (at the beginning and end of the 3-month study period), and the participants were encouraged to weigh themselves every day at home. The observed body weight change in the intervention group was $-2.48 \mathrm{~kg}$ (95\% CI -3.01 to $-1.95 \mathrm{~kg}$ ), with more than half losing $\geq 3 \%$ and about a quarter losing $\geq 5 \%$ of their baseline weight. The proportion of target weight loss achievement was significantly higher $(p<0.05)$ in the intervention group than the control group $(16.7 \%$ for $\geq 3 \%$ weight loss and $5.6 \%$ for $\geq 5 \%$ weight loss). In the Japanese nationwide interventional program for targeting metabolic syndrome, a reduction of body weight of $3 \%-5 \%$ is considered a feasible weight loss target $[3,30]$. The minimum weight reduction required to improve obesity-related risk factors is reported to be $3 \%$ [30]. Logistic regression analysis showed that being assigned to the intervention group was the only predictor of $\geq 5 \%$ weight loss $(p<0.05$ ). For $\geq 3 \%$ weight loss, a higher self-efficacy for activity behavior and a lower baseline MVPA level were also significant predictors $(p<0.05)$. These inconsistencies prevented a strong conclusion. Nevertheless, baseline characteristics of self-efficacy and physical activity may be associated with modest weight loss.

The major strength of our study was the research design of a parallel-group RCT. The findings are highly reliable. A single motivational lecture could be given in various ways, including as a public program, by video distribution with storage media, or online. Further investigations are needed to apply these methods to other local or workplace populations, and future economic analyses will determine whether this approach is indeed cost-effective.

Some limitations were present in our study. First, the study population may have been subject to selection bias because there were more women, some participants were more willing to be involved in the study, and the population included only Japanese people with the majority experiencing grade 1 obesity. These factors limit the generalizability of the study. Second, the study period was relatively short, which means that the results cannot be used to predict the long-term effectiveness or sustainability of any benefits obtained. Third, the single 
Nakata et al.: Effect of a Single Motivational Lecture on Weight Loss

motivational lecture was given by an experienced researcher. Standardization of the lecture would be needed to disseminate the information to the public. Finally, we calculated the sample size based on the effect size from our previous RCT [7] and not based on the minimally clinically significant difference between groups. The default difference of $1.5 \mathrm{~kg}$ was equivalent to about $2 \%$ of baseline weight. Therefore, a single lecture might not be adequate to acquire clinically significant (3\%-5\%) weight loss.

In conclusion, our 3-month RCT tested whether a single motivational lecture could promote weight loss for overweight Japanese adults. Our results suggest that a single lecture can be a cost-effective option to promote modest weight loss in the short term.

\section{Acknowledgement}

We wish to thank the participants in this study and Yuriko Sakairi for her cooperation. Editage provided editorial support in the form of medical writing.

\section{Statement of Ethics}

Participants gave their written informed consent. The study protocol was approved by the institutional review board of the University of Tsukuba Faculty of Medicine (approval No. 739) on 28 February 2013.

\section{Disclosure Statement}

The authors have no conflicts of interest to declare.

\section{Funding Sources}

This study was supported by the Japan Agriculture Ibaraki Public Welfare Federation.

\section{Author Contributions}

Study concept and design: Y.N. and K.H. Intervention: Y.N. Data acquisition:Y.N., H.S., T.T., and H.K. Statistical analysis, interpretation, and writing of the first draft: Y.N. and H.S. Study physician: H.K. Overall supervision as principal investigator: Y.N. All authors contributed to revision of the manuscript and agreed to be accountable for all aspects of this work.

\section{References}

1 NCD Risk Factor Collaboration (NCD-RisC). Trends in adult body-mass index in 200 countries from 1975 to 2014: a pooled analysis of 1698 population-based measurement studies with $19 \cdot 2$ million participants. Lancet. 2016 Apr;387(10026):1377-96.

2 Nishi N. Monitoring Obesity Trends in Health Japan 21. J Nutr Sci Vitaminol (Tokyo). 2015;61 Suppl:S17-9.

3 Tsushita K, S Hosler A, Miura K, Ito Y, Fukuda T, Kitamura A, et al. Rationale and descriptive analysis of specific health guidance: the nationwide lifestyle intervention program targeting metabolic syndrome in Japan. J Atheroscler Thromb. 2018 Apr;25(4):308-22.

4 Aveyard P, Lewis A, Tearne S, Hood K, Christian-Brown A, Adab P, et al. Screening and brief intervention for obesity in primary care: a parallel, two-arm, randomised trial. Lancet. 2016 Nov;388(10059):2492-500. 
5 Beeken RJ, Leurent B, Vickerstaff V, Wilson R, Croker H, Morris S, et al. A brief intervention for weight control based on habit-formation theory delivered through primary care: results from a randomised controlled trial. Int J Obes. 2017 Feb;41(2):246-54.

6 Johns DJ, Hartmann-Boyce J, Jebb SA, Aveyard P; Behavioural Weight Management Review Group. Weight change among people randomized to minimal intervention control groups in weight loss trials. Obesity (Silver Spring). 2016 Apr;24(4):772-80.

7 Nakata Y, Okada M, Hashimoto K, Harada Y, Sone H, Tanaka K. Comparison of education-only versus groupbased intervention in promoting weight loss: a randomised controlled trial. Obes Facts. 2011;4(3):222-8.

8 Nakata Y, Okada M, Hashimoto K, Harada Y, Sone H, Tanaka K. Weight loss maintenance for 2 years after a 6-month randomised controlled trial comparing education-only and group-based support in Japanese adults. Obes Facts. 2014;7(6):376-87.

9 American Diabetes Association and National Institute of Diabetes, Digestive and Kidney Diseases. The prevention or delay of type 2 diabetes. Diabetes Care. 2002 Apr;25(4):742-9.

10 Glasgow RE, Lichtenstein E, Marcus AC. Why don't we see more translation of health promotion research to practice? Rethinking the efficacy-to-effectiveness transition. Am J Public Health. 2003 Aug; 93(8):1261-7.

11 Heshka S, Anderson JW, Atkinson RL, Greenway FL, Hill JO, Phinney SD, et al. Weight loss with self-help compared with a structured commercial program: a randomized trial. JAMA. 2003 Apr;289(14):1792-8.

12 Schulz KF, Altman DG, Moher D; CONSORT Group. CONSORT 2010 statement: updated guidelines for reporting parallel group randomised trials. BMJ. 2010 Mar;340:c332.

13 Examination Committee of Criteria for 'Obesity Disease' in Japan; Japan Society for the Study of Obesity. New criteria for 'obesity disease' in Japan. Circ J. 2002 Nov;66(11):987-92.

14 Hiuge-Shimizu A, Kishida K, Funahashi T, Ishizaka Y, Oka R, Okada M, et al. Absolute value of visceral fat area measured on computed tomography scans and obesity-related cardiovascular risk factors in large-scale Japanese general population (the VACATION-J study). Ann Med. 2012 Feb;44(1):82-92.

15 Nakata Y, Okura T, Matsuo T, Tanaka K. Factors alleviating metabolic syndrome via diet-induced weight loss with or without exercise in overweight Japanese women. Prev Med. 2009 Apr;48(4):351-6.

16 Nakamura Y, Yamamoto T, Okamura T, Kadowaki T, Hayakawa T, Kita Y, et al.; NIPPON DATA 80 Research Group. Combined cardiovascular risk factors and outcome: NIPPON DATA80, 1980-1994. Circ J. 2006 Aug; 70(8):960-4.

17 Kadota A, Hozawa A, Okamura T, Kadowak T, Nakmaura K, Murakami Y, et al.; NIPPON DATA Research Group. Relationship between metabolic risk factor clustering and cardiovascular mortality stratified by high blood glucose and obesity: NIPPON DATA90, 1990-2000. Diabetes Care. 2007 Jun;30(6):1533-8.

18 Iso H, Sato S, Kitamura A, Imano H, Kiyama M, Yamagishi K, et al. Metabolic syndrome and the risk of ischemic heart disease and stroke among Japanese men and women. Stroke. 2007 Jun;38(6):1744-51.

19 Eto M, Ohkawara K, Sasai H, Tsujimoto T, So R, Matsuo T, et al. Efficiency of a free-living physical activity promotion program following diet modification for fat loss in Japanese obese men. J Nutr Sci Vitaminol (Tokyo). 2012;58(6):384-92.

20 Tanaka K, Shigematsu R, Henwood T, Sasai H. Exercise, diet, and weight loss. J Phys Fit Sports Med. 2012;1(3): 457-65.

21 Okura T, Nakata Y, Tanaka K. Effects of exercise intensity on physical fitness and risk factors for coronary heart disease. Obes Res. 2003 Sep;11(9):1131-9.

22 Prochaska JO, Velicer WF. The transtheoretical model of health behavior change. Am J Health Promot. 1997 Sep-Oct;12(1):38-48.

23 Latimer L, Walker LO, Kim S, Pasch KE, Sterling BS. Self-efficacy scale for weight loss among multi-ethnic women of lower income: a psychometric evaluation. J Nutr Educ Behav. 2011 Jul-Aug;43(4):279-83.

24 Sasaki S, Yanagibori R, Amano K. Self-administered diet history questionnaire developed for health education: a relative validation of the test-version by comparison with 3-day diet record in women. J Epidemiol. 1998 Oct;8(4):203-15.

25 Ohkawara K, Oshima Y, Hikihara Y, Ishikawa-Takata K, Tabata I, Tanaka S. Real-time estimation of daily physical activity intensity by a triaxial accelerometer and a gravity-removal classification algorithm. Br J Nutr. 2011 Jun;105(11):1681-91.

26 Oshima Y, Kawaguchi K, Tanaka S, Ohkawara K, Hikihara Y, Ishikawa-Takata K, et al. Classifying household and locomotive activities using a triaxial accelerometer. Gait Posture. 2010 Mar;31(3):370-4.

27 Troiano RP, Berrigan D, Dodd KW, Mâsse LC, Tilert T, McDowell M. Physical activity in the United States measured by accelerometer. Med Sci Sports Exerc. 2008 Jan;40(1):181-8.

28 Mâsse LC, Fuemmeler BF, Anderson CB, Matthews CE, Trost SG, Catellier DJ, etal. Accelerometer data reduction: a comparison of four reduction algorithms on select outcome variables. Med Sci Sports Exerc. 2005 Nov;37(11 Suppl):S544-54.

29 Jolly K, Lewis A, Beach J, Denley J, Adab P, Deeks JJ, et al. Comparison of range of commercial or primary care led weight reduction programmes with minimal intervention control for weight loss in obesity: lighten Up randomised controlled trial. BMJ. 2011 Nov;343:d6500.

30 Muramoto A, Matsushita M, Kato A, Yamamoto N, Koike G, Nakamura M, et al. Three percent weight reduction is the minimum requirement to improve health hazards in obese and overweight people in Japan. Obes Res Clin Pract. 2014 Sep-Oct;8(5):e466-75. 